\section{Anorexia nervosa: is in-patient refeeding necessary?}

SIR: I read with interest the article by Crisp et al (Journal, September 1991, 159, 325-333), who found that outcome for anorexia nervosa in a specialist eating disorders unit was the same if the patients were managed entirely as out-patients as it was if they were admitted for refeeding first. Despite this important finding, which is similar to that of Freeman et al (1991), the authors did not discuss its implications for the management of anorexia nervosa, and I would like to offer the following comments.

The out-patient treatments had a number of clear benefits in that they were more acceptable to the patients, with only four non-compliers out of 40 outpatients, compared with 12 out of 30 in-patients; they were cheaper; and the individual out-patient treatment group had a significantly better socioeconomic outcome. Importantly, there was no difference in the mortality in either treatment setting, the only death occurring in a patient on the waiting list for treatment.

In addition, it is likely the morale of staff engaged in out-patient treatments would be higher, and splitting within the therapeutic team less, as a consequence of not regularly implementing a stressful regime of coercive refeeding and restriction of patient's rights.

In conclusion, it would seem that the further development of such cost-effective treatment models might provide a realistic tool to correct the undoubted 'nationwide paucity of specialised services for anorexia nervosa' commented on by the authors. Such services would still need the backup of some in-patient beds (both medical and psychiatric) for the management of specific complications, such as acute physical collapse, or concurrent suicidal depression, but not routinely for lengthy in-patient refeeding. It is regrettable that the current restrictions on cross charging for out-patient psychiatric treatments may pose a financial disincentive to this process.

Freeman, C. P. L., Shapiro, C., Engleman, H. et al (1991) A randomised controlled trial of the treatment of anorexia nervosa. In Abstract Book, Eating Disorders International Symposium, p. 135. Paris: World Psychiatric Association.

\section{The Gordon Hospital \\ Bloomburg Street \\ London SWIV 2RH}

MARK L. TAtTersall

\section{Anorexia nervosa: is conflict hypothesis valid?}

SIR: I read with interest the comments of Bendall et al (Journal, September 1991, 159, 441) on eating disorders in Asian girls.
A very interesting speculation has arisen in the literature that eating disorders in Asian girls living in Britain may result from intrafamilial conflict. This has come from the observation of different intergenerational value systems in Asian parents settled in Britain and their offspring who have been born and brought up in Britain. But whether such differences can be implicated as causative in anorexia nervosa is a difficult proposition to support.

The psychiatric fraternity tends to look into the negative aspects of family interaction (Hatfield et al, 1987). Conflict hypothesis dominates our thinking. When formulating aetiology of a psychiatric disorder, ascertaining an area of conflict in our patient is often gratifying and we tend to get overwhelmed by this discovery. This domination is often to such an extent that we choose to overlook several other aspects in the individual's illness presentation. The issues of autonomy and control which have become common jargon in other psychiatric illnesses, have also been implicated in anorexia nervosa (Bryant-Waugh \& Lask, 1991).

Whether family difficulties, conflict between traditional family attitudes and Western ones, or intergenerational value differences can be implicated in the genesis of this disorder, has not yet been established. Moreover, this may only be an associated artefact which is being picked up because it is specifically looked for. On the other hand, a more acceptable formulation would be of a young adolescent who chooses to accept the culture of her peer group instead of the culture she inherited, the peer group culture being more influential during adolescence (which is also the common age of presentation of anorexia nervosa). Conflict between cultures may not be the necessary ingredient; adoption of a different (new) cultural value system may make them vulnerable to suffer from this disorder. This would be in line with the suggestion that anorexia nervosa may be a culture-bound syndrome of the Western culture, as discussed elsewhere (Arya, 1991).

The speculation of conflict hypothesis in anorexia nervosa may distract us from the actual conceptual issues involved in the disorder of anorexia nervosa which should be an issue of actual concern. The primary pathology in anorexia nervosa is an overvalued idea or even a delusion of being obese; a distortion of body image. If a neurotic-psychotic continuum is of any value in conceptualising a disorder, anorexia nervosa will tend to weigh rather heavily on the psychotic dimension. As is the case with other psychiatric illnesses, we need to explore other aetiological formulations in biological, social and psychological fields and not get carried away by conflict hypothesis, since convincing answers are 